\title{
GSTT1 and GSTM1 polymorphisms and prostate cancer risk in Asians: a systematic review and meta-analysis
}

\author{
Dajun Liu • Ying Liu • Limei Ran • Huiping Shang • \\ Detian Li \\ Received: 6 March 2013 / Accepted: 26 March 2013 / Published online: 28 June 2013 \\ (C) The Author(s) 2013. This article is published with open access at Springerlink.com
}

\begin{abstract}
Glutathione S-transferases (GSTs) enzymes are involved in conjugation of electrophilic compounds to glutathione, and glutathione S-transferase T 1 (GSTT1) and glutathione S-transferase M 1 (GSTM1) polymorphisms have been implicated as risk factors for prostate cancer. We conducted a systematic review and meta-analysis to define the effect of GSTM1 and GSTT1 null genotypes on prostate cancer risk in Asians. We searched the PubMed and Wanfang Medical databases to identify published case-control studies investigating the associations of GSTM1 and GSTT1 null genotypes with risk of prostate cancer in Asians. Heterogeneity was assessed using Cochran's $Q$ statistic and odds ratios (OR) with corresponding $95 \%$ confidence intervals ( $95 \% \mathrm{CI}$ ) from individual studies were pooled using fixed or random effects models according to the heterogeneity. There were 18 studies (2,046 cases, 2,876 controls) on GSTM1 polymorphism, 15 studies (1,677 cases, 2,431 controls) on GSTT1 polymorphism, and 6 studies (675 cases, 853 controls) on GSTM1/GSTT1 interaction analysis. Overall, GSTM1 null genotype was significantly associated with increased risk of prostate cancer in Asians (random effects OR 1.80, $95 \%$ CI $1.48-2.18, P<0.001$ ), and GSTT1 null genotype was also significantly associated with increased risk of prostate cancer in Asians (random effects OR 1.40, $95 \%$ CI 1.10-1.80, $P<0.001$ ). In addition, the GSTM1/GSTT dual null genotype
\end{abstract}

D. Liu $(\bowtie) \cdot$ H. Shang $\cdot$ D. Li

Department of Kidney, Shengjing Hospital of China

Medical University, NO19 Huaxiang Road, Tiexi District,

Shenyang City 110022, Liaoning Province, China

e-mail: dajunliuliao@yahoo.cn

Y. Liu

Department of Cardiology, Liaoning Province Hospital,

Shenyang City 10016, Liaoning Province, China

L. Ran

Outpatient Department, Affiliated Hospital of Guiyang Medical

College, Guiyang City 550004, Guizhou Province, China was associated with higher risk of prostate cancer in Asians (random effects OR 2.14, $95 \%$ CI 1.59-2.89, $P=0.007$ ). In conclusion, GSTM1 and GSTT1 null genotypes are associated with increased risk of prostate cancer in Asians, and GSTM1 and GSTT1 null genotypes are risk factors for the development of prostate cancer.

Keywords GSTT1 · GSTM1 · Polymorphisms · Prostate cancer

\section{Introduction}

Prostate cancer is a multifactorial disease, and its etiology in detail is still not understood [1,2]. Previous studies have suggested that both environmental and genetic factors are involved in the carcinogenesis of prostate cancer, and individual difference in the susceptibility to carcinogens plays an important role in the development of prostate cancer [3, 4]. The biochemical basis for the individual difference in the susceptibility to carcinogens may be attributed to the genetic polymorphisms of genes implicated in the metabolic detoxification of environmental carcinogens [3, 4]. Currently, there are several important genes encoding enzymes involved in the biotransformation of carcinogens, including glutathione S-transferases (GSTs) enzymes [5, 6]. The GSTs enzymes are involved in the metabolism of a wide variety of potential carcinogens in conjugation of electrophilic compounds to glutathione, and high level of GSTs enzymes is important to protect DNA from damages by detoxifying the electrophilic carcinogens $[5,6]$. The expression of GSTs enzymes is mainly affected by the genetic polymorphisms in the GSTs genes [7, 8]. Previous studies have suggested that individuals with null genotypes of glutathione Stransferase M 1 (GSTM1) and glutathione S-transferase $\mathrm{T}$ 1 (GSTT1) may be unable to eliminate electrophilic carcinogens efficiently and suffer from high risk of cancer [9]. 
Therefore, it has been proposed that GSTT1 and GSTM1 polymorphisms are risk factors for prostate cancer [10-12]. However, the results from previous published studies in Asians reported inconclusive findings [10-19]. Therefore, we conducted a systematic review and meta-analysis to define the effect of GSTM1 and GSTT1 null genotypes on prostate cancer risk in Asians.

\section{Material and methods}

Study identification and eligibility criteria

We searched the PubMed and Wanfang Medical databases to identify published case-control studies investigating the associations of GSTM1 and GSTT1 null genotypes with risk of prostate cancer in Asians. Computer searches were conducted using relevant keywords ("GSTT1," "GSTM1," or "glutathione S-transferase") in combination with keywords ("prostate cancer" and "prostate carcinoma"). There was no language limitation. The reference lists of all relevant studies and review articles were also scanned for other eligible studies. Case-control studies that were performed to define the effect of GSTM1 and GSTT1 null genotypes on prostate cancer risk in Asians were eligible for inclusion. Family-based studies were excluded owing to different design.

\section{Data extraction}

The following information was abstracted from each study: first author, year of publication, study design, matching factors, geographical locations, ethnicity of participants, the definitions and numbers of cases and controls, source of controls, genotyping methods, frequencies of GSTM1/GSTT1 null genotypes, and normal genotypes.

\section{Evidence synthesis}

All associations were assessed using odds ratios (OR) with the corresponding $95 \%$ confidence interval $(95 \% \mathrm{CI})$. Between-study heterogeneity was assessed using Cochran's $Q$ statistic method [20]. The OR with corresponding $95 \%$ CI from individual studies were pooled using fixed or random effects models according to the heterogeneity. When the $P$ value for Cochran's $Q$ statistic was less than 0.05 , and a significant heterogeneity existed across the included studies, the random effects model (DerSimonian and Laird method) was used for meta-analysis [21], or else the fixed effects model (Mantel-Haenszel method) was used [22]. Sensitivity analysis was further performed by excluding single study in turn to assess the impact of individual study on the pooled estimate. The possibility of publication bias was assessed by visually assessing a funnel plot for asymmetry and then assessed using the Egger's test [23]. Statistical analyses were performed using STATA (version 11/SE, Stata Corp., College Stage Station, Texas).

\section{Results}

Eligible studies

Out of 86 studies retrieved by our initial literature search, 18 studies were considered eligible for this systematic review and were included in the meta-analysis [10-19, 24-31]. There were 18 studies (2,046 cases, 2,876 controls) on GSTM1 polymorphism, 15 studies (1,677 cases, 2,431 controls) on GSTT1 polymorphism, and 6 studies (675 cases, 853 controls) on GSTM1/GSTT1 interaction analysis [10-19, 24-31]. These 18 studies were conducted over a period of 12 years (from 2001 to 2012) [10-19, 24-31]. In general, studies were relatively small, and the mean number of cases was 113.7 (range, 50-208) and the mean number of controls was 159.8 (range, 46-336). Most of these 18 studies matched cases to controls by age and gender. The frequency of the GSTM1 null genotype among controls in these 18 studies ranged from 10.0 to $55.4 \%$, while the frequency of the GSTT1 null genotype among controls in these 18 studies ranged from 11.1 to $53.3 \%$ [10-19, 24-31].

\section{Evidence synthesis}

There was obvious between-study heterogeneity among these included studies in the meta-analyses of the associations between prostate cancer and GSTM1 null genotype $(P<0.001)$ and GSTT1 null genotype $(P<0.001)$, but there was no between-study heterogeneity among these included studies on GSTM1/GSTT1 interaction analysis $(P=0.992)$. Overall, GSTM1 null genotype was significantly associated with increased risk of prostate cancer in Asians (random effects OR $1.80,95 \%$ CI 1.48-2.18, $P<0.001$ ), and GSTT1 null genotype was also significantly associated with increased risk of prostate cancer in Asians (random effects OR 1.40, $95 \% \mathrm{CI}$ $1.10-1.80, P<0.001$ ) (Figs. 1 and 2). In addition, the GSTM1/GSTT dual null genotype was associated with higher risk of prostate cancer in Asians (random effects OR 2.14, $95 \%$ CI 1.59-2.89, $P=0.007$ ) (Fig. 3). Sensitivity analysis by excluding single study in turn showed that there were no obvious changes on the pooled estimates after excluding any single study.

Risk of bias

There was no obvious evidence of asymmetry in the funnel plots of this meta-analysis (figures not shown). In addition, the outcomes from Egger's test further showed statistical 
Study

ID
Weight

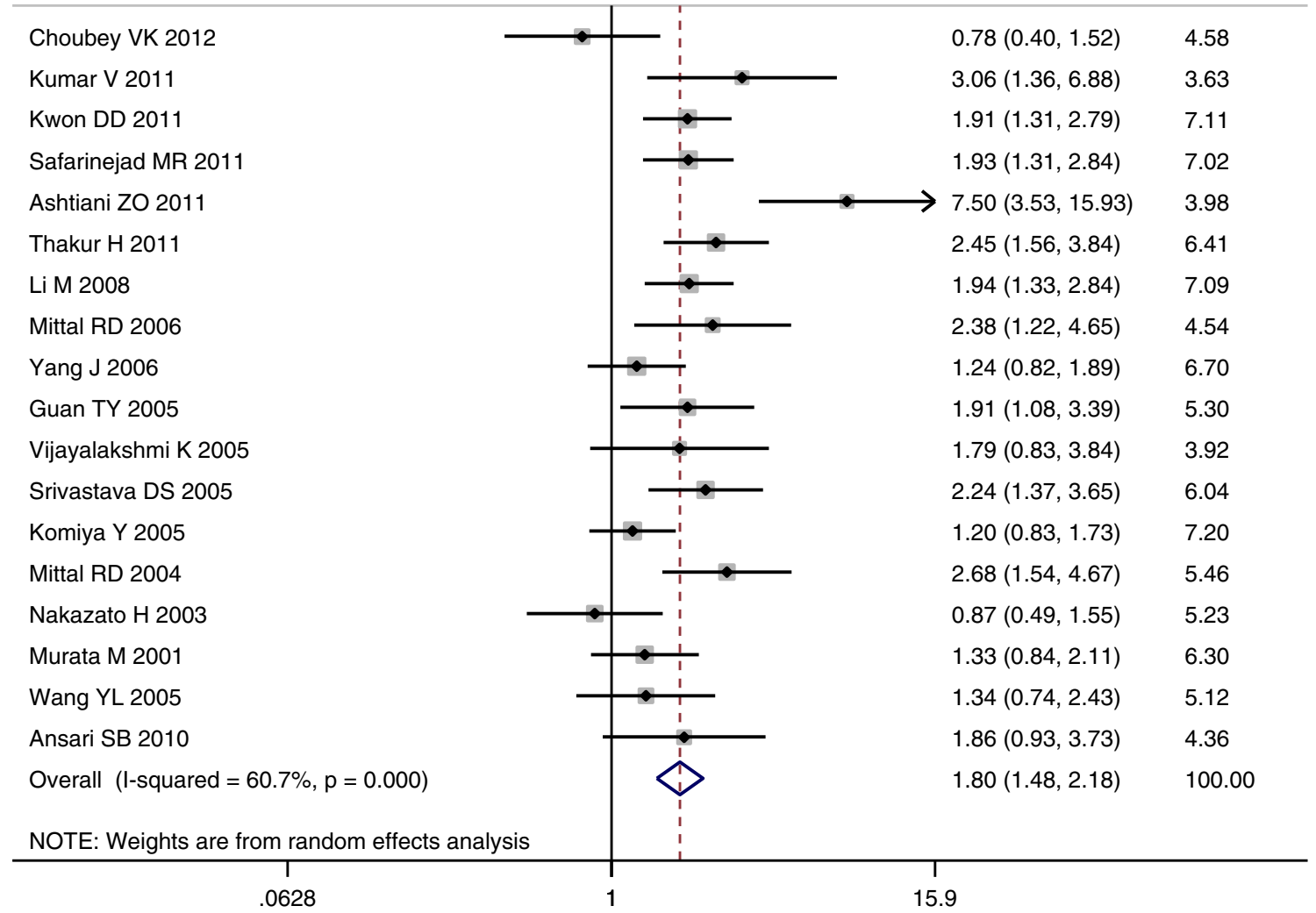

Fig. 1 Forest plot for the meta-analysis of GSTM1 null genotype with prostate cancer risk using random effects model. Each study is shown by the point estimate of the odds ratio (square proportional to the

evidence of symmetry in the funnel plots $\left(P_{\mathrm{GSTM} 1}=0.652\right.$, $\left.P_{\mathrm{GSTT} 1}=0.452, P_{\mathrm{GSTM} 1 / \mathrm{GSTT} 1}=0.327\right)$. Therefore, there was no obvious risk of publication bias in this meta-analysis.

\section{Discussion}

GSTs are members of biotransformation enzymes, and they are phase II enzymes with both catalytic activities and noncatalytic functions [7]. Besides, GSTs are involved in the detoxification of electrophilic compounds by glutathione conjugation, such as carcinogens and cytotoxic drugs [6, 7]. Since the presumed function of GSTs is to protect tissues against toxic and carcinogenic compounds, they are thought to be important determinants in the development of prostate cancer. Previous studies have shown that individuals with GSTM1 null genotype have a decreased capacity to detoxify certain carcinogens, and it is the same with individuals with GSTT1 null genotype [9].

Prostate cancer is a multifactorial disease, and its etiology in detail is still not understood $[4,32,33]$. Previous studies have suggested that both environmental and genetic factors weight of each study) and $95 \%$ confidence interval of the odds ratio (extending lines); the pooled odds ratio and $95 \%$ confidence intervals are depicted as a diamond

are involved in the carcinogenesis of prostate cancer, and individual difference in the susceptibility to carcinogens plays an important role in the development of prostate cancer. The biochemical basis for the individual difference in the susceptibility to carcinogens may be attributed to the genetic polymorphisms of genes implicated in the metabolic detoxification of environmental carcinogens. Previous studies have suggested that individuals with null genotypes of GSTM1 and GSTT1 may be unable to eliminate electrophilic carcinogens efficiently and suffer from high risk of cancer, and it has been proposed that GSTT1 and GSTM1 polymorphisms are risk factors for prostate cancer. However, the results from previous published studies in Asians reported inconclusive findings $[2-4,18,19,26-33]$.

In the present meta-analysis, we conducted the first systematic review and meta-analysis to define the effect of GSTM1 and GSTT1 null genotypes on prostate cancer risk in Asians. There were 18 studies (2,046 cases, 2,876 controls) on GSTM1 polymorphism, 15 studies (1,677 cases, 2,431 controls) on GSTT1 polymorphism, and 6 studies ( 675 cases, 853 controls) on GSTM1/GSTT1 interaction analysis [10-19, 24-31]. Overall, GSTM1 null genotype was significantly 
Study

ID

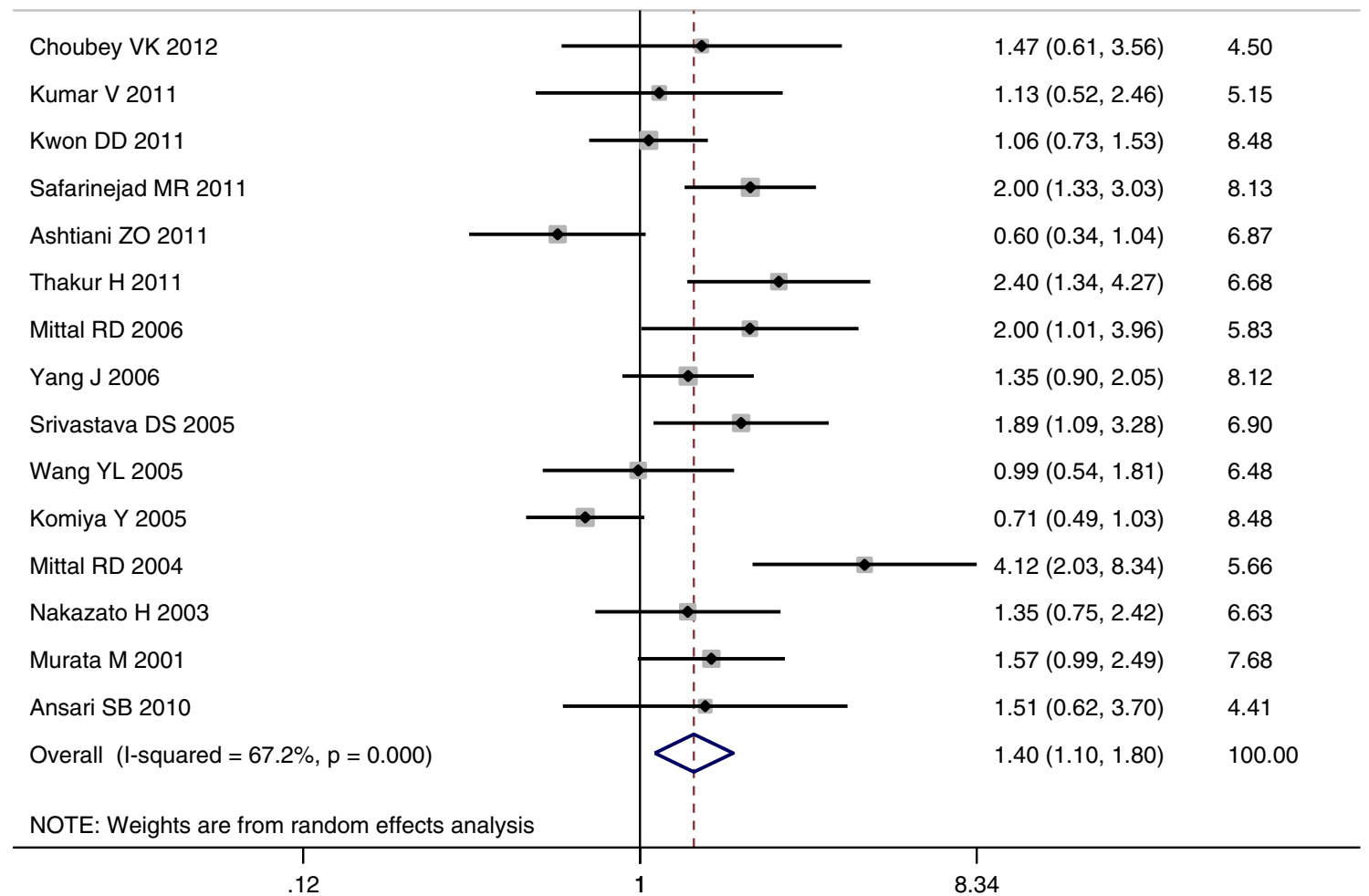

Fig. 2 Forest plot for the meta-analysis of GSTT1 null genotype with prostate cancer risk using random effects model

associated with increased risk of prostate cancer in Asians (Fig. 1), and GSTT1 null genotype was also significantly associated with increased risk of prostate cancer in Asians (Fig. 2). In addition, the GSTM1/GSTT dual null genotype

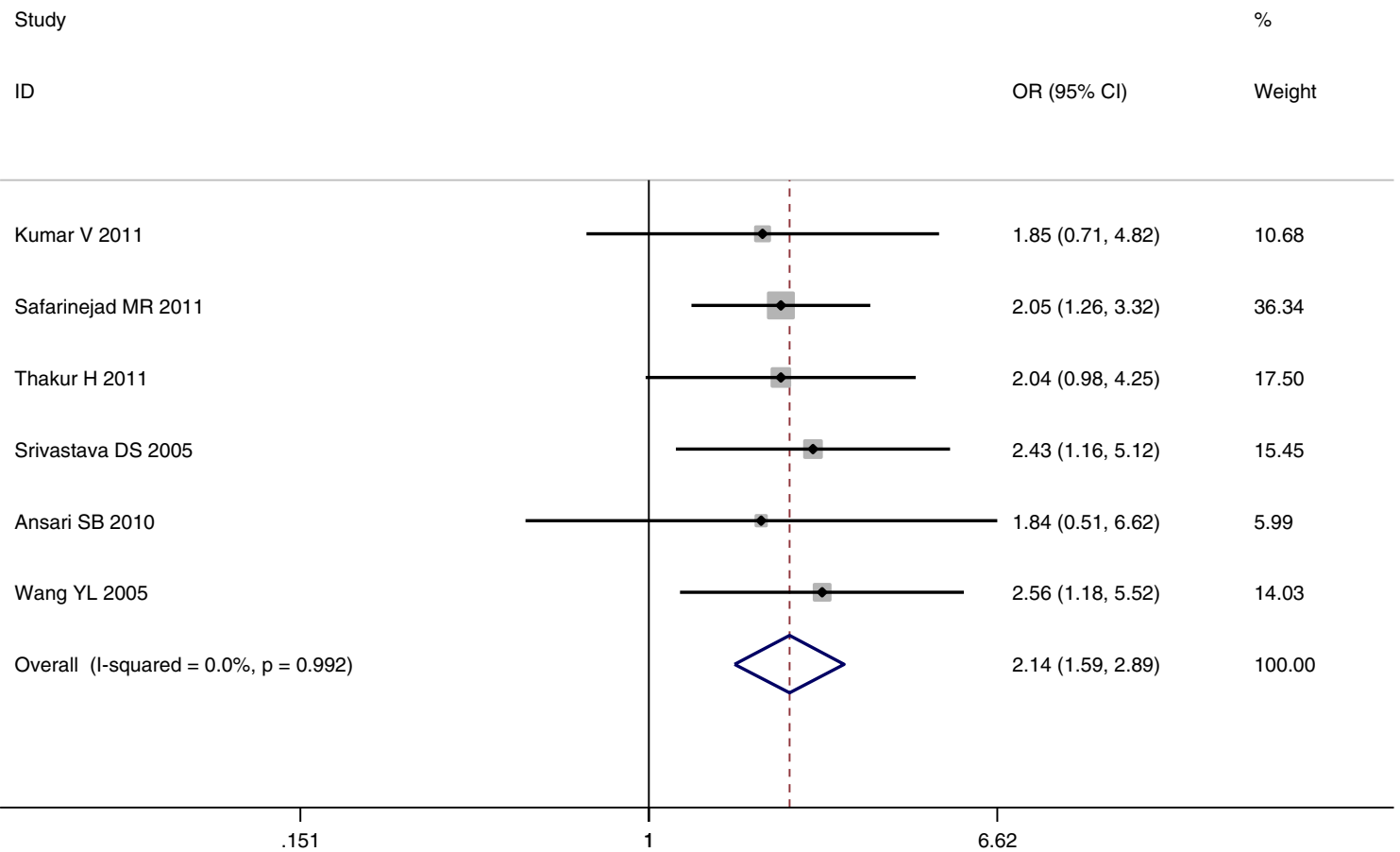

Fig. 3 Forest plot for the meta-analysis of GSTM1/GSTT dual null genotype with prostate cancer risk using fixed effects model 
was associated with higher risk of prostate cancer in Asians (Fig. 3). Therefore, the findings from this meta-analysis showed that GSTM1 and GSTT1 null genotypes are associated with increased risk of prostate cancer in Asians.

This report is the first meta-analysis examining the effect of GSTT1 and GSTM1 polymorphisms on the risk of prostate cancer in Asians. The findings from this meta-analysis also provide evidence for the important roles of GSTs in the development of common cancers. In addition, the GSTT1 and GSTM1 polymorphisms have been proven as risk factors for the development of many kinds of cancers [34-38]. Currently, there are convincing associations between GSTM1 null genotype and risks of colorectal and liver cancer [38, 39]. However, other studies have not confirmed the association between the GSTT1 null genotype and risks of ovarian and oral cancers $[34,36]$. The different associations between GSTT1 and GSTM1 polymorphisms and risk of various kinds of cancers suggest that GSTT1 and GSTM1 polymorphisms may have different roles in the development of cancers.

In conclusion, GSTM1 and GSTT1 null genotypes are associated with increased risk of prostate cancer in Asians, and GSTM1 and GSTT1 null genotypes are risk factors for the development of prostate cancer.

\section{Conflicts of interest None}

Open Access This article is distributed under the terms of the Creative Commons Attribution License which permits any use, distribution, and reproduction in any medium, provided the original author(s) and the source are credited.

\section{References}

1. Jemal A, Bray F, Center MM, Ferlay J, Ward E, Forman D. Global cancer statistics. CA Cancer J Clin. 2011;61:69-90.

2. Green SM, Mostaghel EA, Nelson PS. Androgen action and metabolism in prostate cancer. Mol Cell Endocrinol. 2012;360:3-13.

3. Amankwah EK, Sellers TA, Park JY. Gene variants in the angiogenesis pathway and prostate cancer. Carcinogenesis. 2012;33:1259-69.

4. Pan DL, Samavedi S, Eldefrawy A, Manoharan M. The current status of active surveillance for prostate cancer. Postgrad Med. 2012;124:50-8.

5. Strange RC, Spiteri MA, Ramachandran S, Fryer AA. GlutathioneS-transferase family of enzymes. Mutat Res. 2001;482:21-6.

6. Sharma R, Yang Y, Sharma A, Awasthi S, Awasthi YC. Antioxidant role of glutathione s-transferases: protection against oxidant toxicity and regulation of stress-mediated apoptosis. Antioxid Redox Signal. 2004;6:289-300.

7. Hayes JD, Flanagan JU, Jowsey IR. Glutathione transferases. Annu Rev Pharmacol Toxicol. 2005;45:51-88.

8. Oakley A. Glutathione transferases: a structural perspective. Drug Metab Rev. 2011;43:138-51.

9. Hayes JD, Strange RC. Glutathione S-transferase polymorphisms and their biological consequences. Pharmacology. 2000;61:154-66.

10. Murata M, Watanabe M, Yamanaka M, Kubota Y, Ito H, Nagao M, et al. Genetic polymorphisms in cytochrome P450 (CYP) 1A1, CYP1A2, CYP2E1, glutathione S-transferase (GST) M1 and
GSTT1 and susceptibility to prostate cancer in the Japanese population. Cancer Lett. 2001;165:171-7.

11. Nakazato H, Suzuki K, Matsui H, Koike H, Okugi H, Ohtake N, et al. Association of genetic polymorphisms of glutathione-S-transferase genes (GSTM1, GSTT1 and GSTP1) with familial prostate cancer risk in a Japanese population. Anticancer Res. 2003;23:2897-902.

12. Mittal RD, Srivastava DS, Mandhani A, Kumar A, Mittal B. Polymorphism of GSTM1 and GSTT1 genes in prostate cancer: a study from North India. Indian J Cancer. 2004;41:115-9.

13. Komiya Y, Tsukino H, Nakao H, Kuroda Y, Imai H, Katoh T. Human glutathione S-transferase A1, T1, M1, and P1 polymorphisms and susceptibility to prostate cancer in the Japanese population. J Cancer Res Clin Oncol. 2005;131:238-42.

14. Srivastava DS, Mandhani A, Mittal B, Mittal RD. Genetic polymorphism of glutathione S-transferase genes (GSTM1, GSTT1 and GSTP1) and susceptibility to prostate cancer in Northern India. BJU Int. 2005;95:170-3.

15. Vijayalakshmi K, Vettriselvi V, Krishnan M, Shroff S, Vishwanathan KN, Jayanth VR, et al. Polymorphisms at GSTM1 and GSTP1 gene loci and risk of prostate cancer in a South Indian population. Asian Pac J Cancer Prev. 2005;6:309-14.

16. Mittal RD, Mishra DK, Mandhani A. Evaluating polymorphic status of glutathione-S-transferase genes in blood and tissue samples of prostate cancer patients. Asian Pac J Cancer Prev. 2006;7:444-6.

17. Yang J, Wu HF, Zhang W, Gu M, Hua LX, Sui YG, et al. Polymorphisms of metabolic enzyme genes, living habits and prostate cancer susceptibility. Front Biosci. 2006;11:2052-60.

18. Kumar V, Yadav CS, Datta SK, Singh S, Ahmed RS, Goel S, et al. Association of GSTM1 and GSTT1 polymorphism with lipid peroxidation in benign prostate hyperplasia and prostate cancer: a pilot study. Dis Markers. 2011;30:163-9.

19. Choubey VK, Sankhwar SN, Tewari R, Sankhwar P, Singh BP, Rajender S. Null genotypes at the GSTM1 and GSTT1 genes and the risk of benign prostatic hyperplasia: a case-control study and a meta-analysis. Prostate. 2013;73:146-52.

20. Cochran WG. The combination of estimates from different experiments. Biometrics. 1954;10:101-29.

21. DerSimonian R, Laird N. Meta-analysis in clinical trials. Control Clin Trials. 1986;7:177-88.

22. Mantel N, Haenszel W. Statistical aspects of the analysis of data from retrospective studies of disease. J Natl Cancer Inst. 1959;22:719-48.

23. Egger M, Davey Smith G, Schneider M, Minder C. Bias in meta-analysis detected by a simple, graphical test. BMJ. 1997;315:629-34.

24. Guan TY, Li M, Na YQ. [Polymorphism of metabolic gene and genetic susceptibility to prostate cancer]. Zhonghua Wai Ke Za Zhi. 2005;43:1467-70.

25. Wang YL, Jiang J, Wang LF, Liu YF. Polymorphisms of glutathione-S-transferase genes GSTM1 and GSTT1 and prostate cancer risk in Chinese population. Acta Academiae Medicinae Militaris Tertiae. 2005;27:1039-41.

26. Li M, Guan TY, Li Y, Na YQ. Polymorphisms of GSTM1 and CYP1A1 genes and their genetic susceptibility to prostate cancer in Chinese men. Chin Med J (Engl). 2008;121:305-8.

27. Ansari SB, Vasudevan R, Bakhshi A, Mirinargesi M, Patimah I, Sabariah AR, et al. Analysis of glutathione S-transferase (M1, T1 and P1) gene polymorphisms in Iranian prostate cancer subjects. Afr J Biotechnol. 2010;9:7230-5.

28. Ashtiani ZO, Hasheminasab SM, Ayati M, Goulian BS, Modarressi MH. Are GSTM1, GSTT1 and CAG repeat length of androgen receptor gene polymorphisms associated with risk of prostate cancer in Iranian patients? Pathol Oncol Res. 2011;17:269-75.

29. Kwon DD, Lee JW, Han DY, Seo IY, Park SC, Jeong HJ, et al. Relationship between the glutathione-S-transferase P1, M1, and T1 
genotypes and prostate cancer risk in Korean subjects. Korean $\mathrm{J}$ Urol. 2011;52:247-52.

30. Safarinejad MR, Shafiei N, Safarinejad SH. Glutathione Stransferase gene polymorphisms (GSTM1, GSTT1, GSTP1) and prostate cancer: a case-control study in Tehran, Iran. Prostate Cancer Prostatic Dis. 2011;14:105-13.

31. Thakur H, Gupta L, Sobti RC, Janmeja AK, Seth A, Singh SK. Association of GSTM1T1 genes with COPD and prostate cancer in north Indian population. Mol Biol Rep. 2011;38:1733-9.

32. Galsky MD, Small AC, Tsao CK, Oh WK. Clinical development of novel therapeutics for castration-resistant prostate cancer: historic challenges and recent successes. CA Cancer J Clin. 2012;62:299-308.

33. Tammela TL. Endocrine prevention and treatment of prostate cancer. Mol Cell Endocrinol. 2012;360:59-67.

34. Economopoulos KP, Sergentanis TN, Vlahos NF. Glutathione Stransferase $\mathrm{M} 1, \mathrm{~T} 1$, and $\mathrm{P} 1$ polymorphisms and ovarian cancer risk: a meta-analysis. Int J Gynecol Cancer. 2010;20:732-7.
35. Gao LB, Pan XM, Li LJ, Liang WB, Bai P, Rao L, et al. Null genotypes of GSTM1 and GSTT1 contribute to risk of cervical neoplasia: an evidence-based meta-analysis. PLoS One. 2011;6:e20157.

36. Zhang ZJ, Hao K, Shi R, Zhao G, Jiang GX, Song Y, et al. Glutathione S-transferase M1 (GSTM1) and glutathione Stransferase T1 (GSTT1) null polymorphisms, smoking, and their interaction in oral cancer: a huge review and meta-analysis. Am J Epidemiol. 2011;173:847-57.

37. Song K, Yi J, Shen X, Cai Y. Genetic polymorphisms of glutathione S-transferase genes GSTM1, GSTT1 and risk of hepatocellular carcinoma. PLoS One. 2012;7:e48924.

38. Wang D, Zhang LM, Zhai JX, Liu DW. GSTM1 and GSTT1 polymorphisms and colorectal cancer risk in Chinese population: a meta-analysis. Int J Colorectal Dis. 2012;27:901-9.

39. Wang B, Huang G, Wang D, Li A, Xu Z, Dong R, et al. Null genotypes of GSTM1 and GSTT1 contribute to hepatocellular carcinoma risk: evidence from an updated meta-analysis. J Hepatol. 2010;53:508-18. 